\title{
A cost-utility analysis of a rehabilitation service for people living with and beyond cancer
}

\author{
Jeff Round ${ }^{1,2^{*}}$, Baptiste Leurent ${ }^{1}$ and Louise Jones ${ }^{1}$
}

\begin{abstract}
Background: We conducted a wait-list control randomised trial of an outpatient rehabilitation service for people living with and beyond cancer, delivered in a hospice day care unit. We report the results of an economic evaluation undertaken using the trial data.
\end{abstract}

Methods: Forty-one participants were recruited into the study. A within-trial stochastic cost-utility analysis was undertaken using Monte-Carlo simulation. The outcome measure for the economic evaluation was quality adjusted life years (QALYs). Costs were measured from the perspective of the NHS and personal social services. Uncertainty in the observed data was captured through probabilistic sensitivity analysis. Scenario analysis was conducted to explore the effects of changing the way QALYs were estimated and adjusting for baseline difference in the population. We also explore assumptions about the length of treatment benefit being maintained.

Results: The incremental cost-effectiveness ratio (ICER) for the base-case analysis was $£ 14,231$ per QALY. When QALYS were assumed to change linearly over time, this increased to $£ 20,514$ per QALY at three months. Adjusting the estimate of QALYs to account for differences in the population at baseline increased the ICER to $£ 94,748$ per QALY at three months. Increasing the assumed length of treatment benefit led to reduced ICERs in all scenarios.

Conclusions: Although the intervention is likely to be cost-effective in some circumstances, there is considerable uncertainty surrounding the decision to implement the service. Further research, informed by a formal value of information analysis, would reduce this uncertainty.

Keywords: Cost-utility, Economics, Health economics, Survivorship, Living beyond cancer

\section{Background}

More than 2 million people across the UK are currently living with and beyond cancer. For those who have recently completed active treatments, but for whom cure remains unlikely, there may be considerable physical and psychological morbidity [1,2]. Increasingly there is a trend towards providing palliative care to those who might still be considering active treatments from oncology services to improve symptom control $[3,4]$. It remains common practice to refer people with cancer to palliative care specialists when standard therapy has failed and cure is no longer likely. At the point of referral estimating prognosis may be difficult [5]. Patients may typically access the service for more than three

\footnotetext{
* Correspondence: j.round@ucl.ac.uk

${ }^{1}$ Marie Curie Palliative Care Research Unit, University College London, London, UK

${ }^{2}$ University College London Comprehensive Clinical Trials Unit, University College London, Gower Street, London WC1E 6BT, UK
}

months and the care that is offered is adjusted according to deteriorating health status. Attendance at day care services may enable a smooth transition to terminal care either as a hospice in patient or at home. A rehabilitation-focussed approach to care aims to improve quality of survival, helping people adapt to current circumstances, lead fulfilling lives and function at a minimum level of dependency regardless of life expectancy [6]. For those with active recurrent progressive disease, many UK hospices provide out-patient specialist palliative day therapy. Such care is increasingly offered to not only to people with cancer but to those with a range of other advanced, progressive diseases who are approaching the end of their lives.

Despite a common focus on provision of individually tailored holistic care, there is variability in the interventions available, ranging from social support to a clinically oriented service [7]. Specialist palliative care provision in 
hospices within the UK is largely dependent on funding spearheaded by the voluntary sector and what is available in any locality will depend on local resources and availability of staff, premises and equipment. More specialised services rely on the availability of a multidisciplinary team with the necessary skills and training. Outpatient clinical assessment may be offered by doctors or clinical nurse specialists. Such consultations may be used to identify particular supportive care needs and to agree a care plan that might include sessions delivered by physiotherapists, counsellors or complementary therapists. In addition, some attendees may benefit from the support of interaction with others who are faced with similar health challenges or from group activities such as relaxation or guidance for the management of symptoms such as shortness of breath [8].

There is little current evidence on the relative effectiveness of different models of day therapy, and little guidance to inform those setting up new services or considering how best to use limited available resource for the maximum benefit of patients $[9,10]$. Recent reports that review available qualitative data suggest a positive impact on quality of life for people receiving hospice day care [11]. However data reporting the effects on quantitative outcomes are scarce, in part due to the complexity of services offered and uncertainty over the choice of appropriate measures [12,13]. Whilst there is some evidence from a study conducted in 5 centres in SE England [14] that those receiving hospice day care used fewer other health care services, there is a lack of research to assess the costs and cost-effectiveness of hospice day care services [15].

Outpatient services provided by hospices as described above are considered by some to be an effective way of providing care to patients that addresses both the physical and psychological needs of patients. We conducted an RCT that showed an outpatient service was capable of addressing these needs. We also investigated whether such a service is cost-effective and this is the focus of the current report. In reporting the clinical results of the trial [6] we were restricted by space to describing a limited economic analysis of the data available at the primary outcome point of three months from randomisation (these results are reproduced here in Table 1). In this study we report in full the methodology and results of the economic evaluation, in the form of a cost utility analysis with full probabilistic sensitivity analysis and scenario analyses.

\section{Methods}

This analysis is based on data collected as part of a completed randomised controlled trial of the effectiveness of a complex rehabilitation intervention in a DTU for people with advanced, progressive, recurrent cancer [16]. Ethical approval for the trial was received from the joint University College London/University College London Hospital (UCLH) Research Ethics Committee on October 5, 2009, ref. 09/H0714/46. The trial was registered with ISRCTN number 22485853. The primary outcome of the trial was psychological subscale of the Supportive Care Needs Survey (SCNS) [17].

Data were collected as part of a two-arm, wait-list, randomised controlled trial of the rehabilitation service in addition to usual care compared with usual care alone [16]. Forty-one patients with active, progressive, recurrent malignancies were recruited from breast and haematological oncology out-patient clinics at the Royal Free Hospital (RFH) and University College London Hospital (UCLH) joint cancer centre London UK, between August 2010 and July 2011. Ninety-three per cent were female and the median age was 62 years. Thirty-six participants (20 in the intervention arm, and 16 in the control group) completed the 3-month follow-up for whom both effectiveness and economic outcomes were available. Reasons for drop out were independent of the intervention and analysis was performed on complete data.

\section{The rehabilitation intervention}

The rehabilitation intervention was developed and defined using an iterative "plan-do-study-act" approach consistent with the guidance on developing complex healthcare interventions outlined by the UK Medical Research Council [18]. It offers individual clinical assessment, agreed goal setting and a complex package of specialist multi-disciplinary services including physical and psychological therapies. The aim is to achieve an agreed date for discharge from the service, usually within 3-6 months. Those whose health continues to deteriorate may not achieve discharge. Details of the intervention are described in the form of a written manual, available from the authors.

Table 1 Incremental cost-effectiveness ratio (reproduced from Jones et al., 2012 [16])

\begin{tabular}{|c|c|c|c|c|c|c|}
\hline \multicolumn{2}{|l|}{ Control } & \multicolumn{2}{|l|}{ Intervention } & \multicolumn{3}{|l|}{ Incremental } \\
\hline Cost $(95 \% \mathrm{Cl})$ & QALYs $(95 \% \mathrm{Cl})$ & Cost $(95 \% \mathrm{Cl})$ & QALYs $(95 \% \mathrm{Cl})$ & Cost $(95 \% \mathrm{Cl})$ & QALYs $(95 \% \mathrm{Cl})$ & ICER \\
\hline$£ 1,590$ & 0.11 & $£ 2,544$ & 0.16 & $£ 955$ & 0.05 & $£ 19,391$ \\
\hline$(£ 1,129-£ 2,193)$ & $(0.058-0.157)$ & $(£ 1,890-£ 3,482)$ & $(0.137-0.187)$ & $(£ 82-£ 1,975)$ & $(0.000-0.112)$ & \\
\hline
\end{tabular}




\section{Effectiveness of the intervention}

The effectiveness of this intervention was evaluated in a wait-list randomised controlled trial conducted in a large inner city cancer centre and the results are published elsewhere [16]. For the trial we chose participants with active recurrent progressive breast and haematological cancers because post treatment needs are well documented for these disease sites. We found that the outpatient day-therapy rehabilitation approach was significantly more effective than usual care alone for the primary outcome of psychological needs for care as measured by the Supportive Care Needs Survey [17]. Healthrelated quality of life measured using the EQ-5D was also found to be higher in the treatment group, although this difference was not significant and the trial was not powered to detect a difference on the quality of life outcome measure.

\section{Cost-effectiveness of the intervention}

The trial was limited to a three month data collection phase due to the wait list design, ethical constraints and restrictions on research funding. In this paper we develop more fully the economic evaluation to a) include a full probabilistic sensitivity analysis and b) test assumptions made about treatment benefit in the deterministic analysis. We test these assumptions through scenario analyses focusing on i) the methods used to calculate quality adjusted life years and ii) the extrapolation of treatment costs and benefits beyond the initial three month follow-up period. Our updated results provide additional information on the likely longer term impact of the intervention. This is of importance as people with advanced disease are likely to have on-going supportive care needs and many will remain within the service for an extended period. For those that achieve discharge from the service, it is hoped that any benefits accrued would be maintained.

A cost-utility analysis of the intervention compared with usual care was conducted. The main outcome of interest for the cost-utility analysis was quality of life, expressed as quality adjusted life years (QALYs) and measured using the EQ-5D. The EQ-5D is a commonly used instrument for the measurement of health related quality of life [19] and is recommended for use in technology appraisal by the National Institute for Health and Care Excellence [20]. Although prognosis of participants was limited, minimum dependency and maximum function were key components of the primary outcome and we therefore considered the EQ-5D to be the most appropriate preference based measure of HRQoL [21]. The analysis was undertaken from both an NHS and a personal social services perspective. Results are reported as incremental cost-effectiveness ratios (ICERs) and cost-effectiveness acceptability curves (CEACs). A CEAC shows the percentage of simulations in which an intervention is cost-effective (based on the results of the Monte Carlo simulations) across a range of willingness to pay per QALY. Costs and benefits were estimated for the three month trial period in the analysis, thus no discount rate was applied. Prices are given in UK pounds at 2010-2011 values.

The base-case analysis presented is a stochastic costeffectiveness analysis [22]. A Monte Carlo process modelling 10,000 simulations was performed following the approach established by O'Hagan and colleagues [23-25]. Parameter uncertainty was reflected in the analysis through the use of probabilistic sensitivity analysis (PSA) in a Bayesian framework. Due to the limited data available, an uninformative prior was assumed [26]. PSA is used to reflect the uncertainty inherent in input parameters estimated from samples of populations. Model parameters are characterised not as point estimates based on measures of central tendency but as probability distributions. For each simulation in a PSA, an estimate for each parameter is drawn from the assigned distribution. The individual values sampled from the distributions are then used to estimate the results of the model for that simulation. The results of all simulations are then combined to give an overall result. Details of the mean values and probability distributions estimated for each parameter are presented in Table 2.

At three months, participants reported their use of medical services since baseline using a modified version of the Client Service Receipt Inventory CSRI [27]. We collected data primarily on their use of NHS and hospice services. Commonly accessed services included general practitioners, physiotherapists, psychologists or other counsellors, outpatient hospital appointments. Other services were less frequently accessed and so have been aggregated in the analysis, weighted according to frequency of access and cost (see Table 2).

Data on costs were derived from the Unit Costs of Health and Social Care [28]; where publicly available data were not available, unit costs were obtained from the hospice where the trial was conducted. The primary source for unit cost data [28] does not provide estimates of variability in unit costs. To reflect variation in unit costs, an adjustment is made to the total cost estimated for each simulation using the market forces factor used to calculate local adjustments to NHS tariff prices. An upper and lower bound for a uniform distribution was defined to reflect the market forces factor [29]. For each simulation, a sample from this distribution was used to weight the expected total cost per patient. Sources for all unit costs are presented in Table 3. The way in which variability in costs was accounted for in the model is the underlying reason that the results presented in Jones et al. [16] differ from the base-case results presented here. In our previous estimate, costs were not included 
Table 2 Resource use and utility estimates used in economic evaluation (estimated from trial data)

\begin{tabular}{|c|c|c|c|c|c|c|}
\hline & Control & & & Intervention & & \\
\hline & Mean $\left(s^{2}\right)$ & Distribution & (alpha, beta) & Mean $\left(\mathrm{s}^{2}\right)$ & Distribution & (alpha, beta) \\
\hline Utility values & $0.447(0.01)$ & Gamma & $(29.45,0.02)$ & $0.654(0.002)$ & Gamma & $(48.01,0.01)$ \\
\hline Outpatient appointment & $5.75(1.00)$ & Gamma & $(32.95,0.17)$ & $5.16(0.44)$ & Gamma & $(61.08,0.08)$ \\
\hline GP appointment & $1.56(0.25)$ & Gamma & $(9.65,0.16)$ & $1.00(0.10)$ & Gamma & $(10.50,0.10)$ \\
\hline Other NHS services & $1.75(0.39)$ & Gamma & $(7.85,0.22)$ & $0.32(0.03)$ & Gamma & $(3.90,0.08)$ \\
\hline Counsellor & $0.94(0.34)$ & Gamma & $(2.56,0.37)$ & Not applicable & Not applicable & Not applicable \\
\hline Physiotherapist & $1.81(1.24)$ & Gamma & $(2.65,0.68)$ & Not applicable & Not applicable & Not applicable \\
\hline Outpatient Hospice & Not applicable & Not applicable & Not applicable & $2.05(0.07)$ & Gamma & $(59.26,0.03)$ \\
\hline DTU Attendance & Not applicable & Not applicable & Not applicable & $5.20(1.05)$ & Gamma & $(25.74,0.20)$ \\
\hline Hospice Physiotherapy & Not applicable & Not applicable & Not applicable & $3.55(0.97)$ & Gamma & $(12.98,0.27)$ \\
\hline Other hospice services & Not applicable & Not applicable & Not applicable & $1.5(0.21)$ & Gamma & $(10.82,0.14)$ \\
\hline
\end{tabular}

in the probabilistic sensitivity analysis. We consider the current analysis reported here to be more robust than the previous analysis.

Utility values were estimated using the EQ-5D social tariff for the UK [19] with scores estimated at baseline and at three month follow-up. In the base-case analysis as reported elsewhere [16] QALYs were initially calculated using the a crude mean difference between the intervention and control group at three months. We test this assumption in scenario analyses as described below.

\section{Scenario analyses}

We conducted a series of scenario-based sensitivity analyses to explore variation in our results that arise owing to changes in assumptions made for the base-case analysis. We explored variations around two key assumptions. The first is the manner in which we calculated
QALYs from the trial data. The second assumption we test is the length of time over which benefit is maintained following treatment.

In the analysis reported alongside the clinical trial [16] we used a crude estimate of benefit, where we simply calculated the treatment effect in QALYs based on the difference in utility scores between treatment groups at three months follow-up. This has the effect of allocating all benefit arising from treatment immediately at the start of treatment. While this may be appropriate in some scenarios, for this rehabilitation intervention it is likely that this overstates any differences in QALYs between groups at three months. We therefore explore two alternative approaches to calculating QALYs.

In the first sensitivity scenario QALYs were calculated by integrating utility values over time (area under the curve), based on EQ-5D values at baseline and three

Table 3 Cost estimates used in economic evaluation

\begin{tabular}{|c|c|c|c|c|c|}
\hline Resource & $\begin{array}{l}\text { Mean cost/ } \\
\text { unit }\end{array}$ & Unit & Resource & $\begin{array}{l}\text { Mean cost/ } \\
\text { unit }\end{array}$ & Unit \\
\hline $\begin{array}{l}\text { Outpatient } \\
\text { appointment }\end{array}$ & $£ 152.00[28]$ & Outpatient attendance & Counsellor & $£ 44.00[28]$ & Per hour client contact \\
\hline GP appointment & $£ 32.00[28]$ & $\begin{array}{l}\text { per } 12 \text { minute } \\
\text { consultation }\end{array}$ & Inpatient admission & $£ 225.00[28]$ & NHS cost per bed day \\
\hline GP home visit & $£ 115.00[28]$ & $\begin{array}{l}25 \text { minute visit ( } £ 4.60 \text { per } \\
\text { minute) }\end{array}$ & Outpatient Hospice & $£ 104.40^{*}$ & Per hospice outpatient appt \\
\hline Practice Nurse & $£ 10.00[28]$ & Per consultation & DTU Attendance & $£ 139.80^{*}$ & Cost per DTU attendance \\
\hline $\begin{array}{l}\text { District nurse } \\
\text { (home) }\end{array}$ & $£ 68.00[28]$ & Per hour home visit & Hospice Physiotherapist & $£ 18.50[28]$ & 30 minute consultation \\
\hline $\begin{array}{l}\text { Palliative nurse } \\
\text { (home) }\end{array}$ & $£ 68.00[28]$ & Per hour home visit & Hospice Other & $£ 37.27[28]^{*}$ & $\begin{array}{l}\text { Weighted average of hospice services used } \\
\text { by the intervention group }\end{array}$ \\
\hline $\begin{array}{l}\text { Occupational } \\
\text { therapist }\end{array}$ & $£ 51.33[28]$ & 40 minute consultation & $\begin{array}{l}\text { Control group other } \\
\text { services }\end{array}$ & $£ 66.09[28]^{*}$ & Weighted average of other service use costs \\
\hline Physiotherapist & $£ 18.50[28]$ & 30 minute consultation & $\begin{array}{l}\text { Intervention group other } \\
\text { services }\end{array}$ & $£ 70.42[28]^{*}$ & Weighted average of other service use costs \\
\hline Psychologist & $£ 81.00[28]$ & Per hour client contact & & & \\
\hline
\end{tabular}

${ }^{*}$ Costs provided by the hospice participating in the study. 
months, and assuming a linear change between measurements [30]. This approach assumes that changes in outcome accrue gradually over time. We thereafter refer to this scenario as "area under the curve approach".

We then adjusted the estimate of QALY difference between groups to account for differences at baseline. This second approach takes into account the imbalance between groups in EQ5D at baseline and how this could affect the observed difference in QALYs [30]. It can also increase precision by reducing standard errors of the difference in QALY estimates. However the adjustment is based on a linear regression and assumes a normal distribution of the difference in QALYs between groups, which may not always hold in a small sample. We thereafter refer to this scenario as "baseline adjusted approach".

The final scenario we tested was the length of time over which patients are assumed to derive benefit from the intervention. The base-case analysis considered only the period during which patients were active trial participants. However, an important component of the intervention is that it aims to enable patients to be discharged from the service, or to reduce their frequency of attendance through agreed goal-setting. It is expected that benefit would be maintained for some period of time following discharge. However, the limited time frame of the trial follow-up period prevented the collection of data on patient outcomes following discharge. To test what might happen, we considered the benefit of treatment being maintained over three, six and nine months beyond completion of the follow-up.

Further assumptions were needed about how to estimate benefits at future time points in the absence of follow-up data beyond three months. We have assumed that the mean difference in outcomes at the three month follow-up point was maintained at future time points. Although it is unlikely that in practice benefit would be maintained at a constant level between three month follow-up and one year, there is no evidence on which to base any additional assumption. Our current assumption makes no prediction about changes in quality of life following treatment; rather we assume only that the difference between the treatment and control groups is maintained. We consider the implications of this assumption in more detail in the discussion. We also assume that no additional differences in cost arise during the extended period of analysis. While it is possible that the intervention could influence resource use beyond the three month trial period we expect most of the difference to happened in the first three months when patients were attending the day therapy unit (DTU). It is unknown in which direction any longer term difference would be and we therefore made the assumption of no difference in resource use between arms after the initial three month period.
Figure 1 illustrates how different approaches to estimating patient benefit can lead widely differing results in QALYs used in an analysis.

\section{Results}

\section{Resource use}

In Table 4 we show a summary of the mean cost for the key resource use categories for the control and intervention arms. While the study was not powered to detect differences in resource use, the descriptive results clearly show that the primary difference in expected cost arises from attendance at hospice outpatient and day therapy units. These cost components form part of the intervention and were not available to the control group.

\section{Base-case analysis}

Over the trial period of 3 months, the expected mean difference in cost from the Monte Carlo simulation in the base-case analysis was $£ 735$ (95\% Bayesian credible intervals (CI) $£ 221$ to $£ 1,271$ ) and the mean difference in QALYs was 0.052 (95\% CI 0.040 to 0.063). The ICER of the mean incremental values is $£ 14,231$ per QALY (Table 5).

\section{Scenario analyses}

The results of the scenario analyses are presented in Tables 5 and 6 as well as Figures 1 and 2. Using the area under the curve approach to estimating QALYs then the intervention is expected to be cost-effective in $42.9 \%$ and $64.1 \%$ of simulations at threshold values of $£ 20,000$ and $£ 30,000$ respectively at three months, with an ICER of $£ 20,514$ (Table 6). The likelihood of the intervention being cost-effective increases with the length of time benefit is expected to be maintained. It rises to $77.4 \%$ at a threshold value of $£ 20,000$ when benefit is measured at six months. If benefit is maintained over 12 months then at a threshold value of $£ 20,000$ the intervention is expected to be cost-effective in $89.6 \%$ of simulations (Figure 2).

When we calculate QALYs adjusted for baseline differences in the control and intervention population, the intervention becomes less likely to be cost-effective across all time-horizons and threshold values. The estimated ICER at three months is $£ 94,748$ (Table 7) and consequently the intervention is highly unlikely to be cost-effective when benefit is only gained for three months, with a probability of being cost-effective of just $3.7 \%$ at the $£ 20,000$ threshold and $9.5 \%$ at the higher $£ 30,000$ threshold (Figure 3 ). If benefit is maintained for a total of six months then the intervention reaches a probability of being cost effective of $29.5 \%$ and $45.2 \%$ as threshold values of $£ 20,000$ and $£ 30,000$. Only where benefit is maintained for nine months or longer is the intervention more likely than not to be cost-effective at 

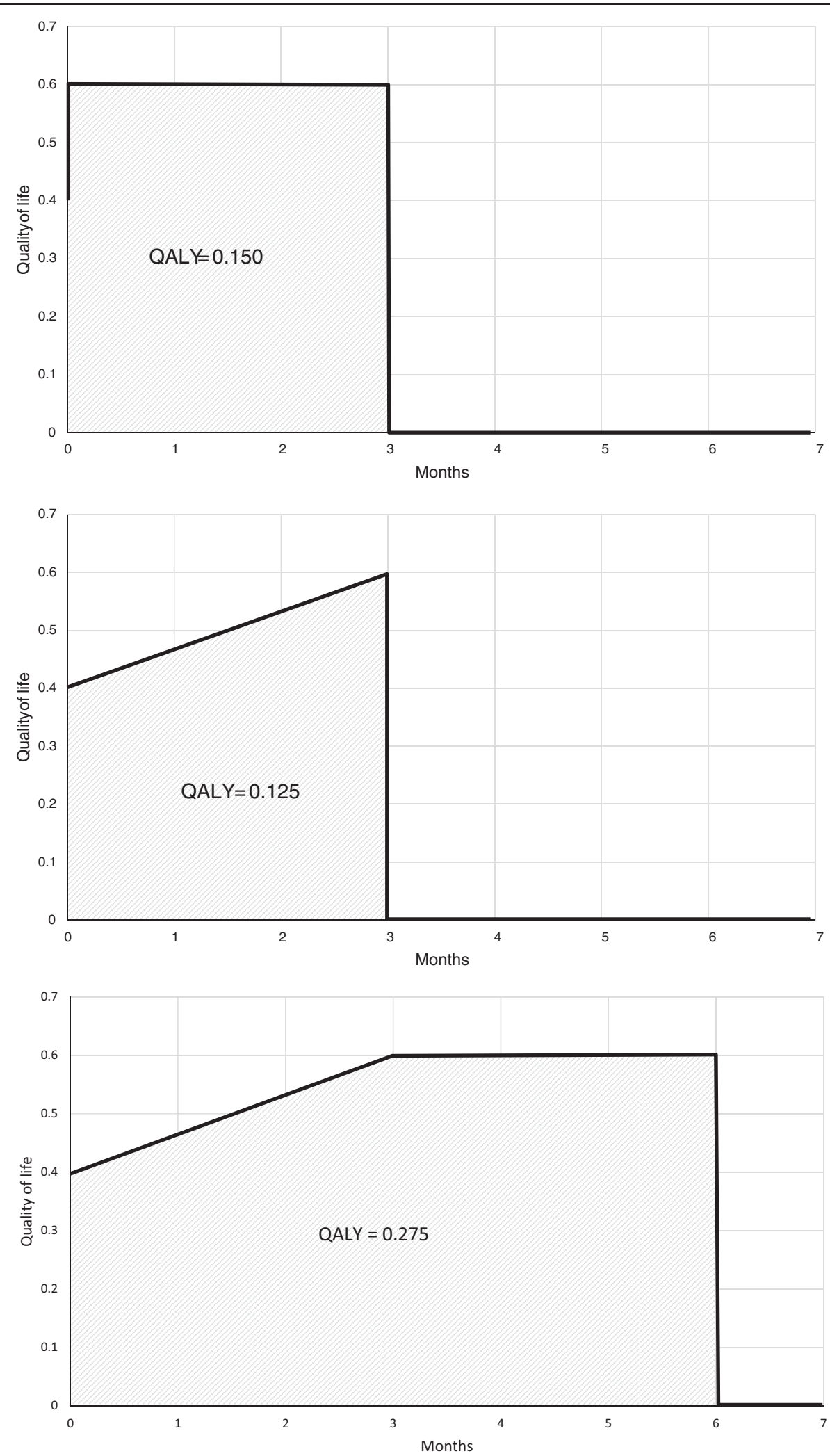

Figure 1 Example of different approaches for calculating QALYs. 
Table 4 Deterministic mean cost of individual resources

\begin{tabular}{lll}
\hline & Expected cost \\
\cline { 2 - 3 } Resource & Control & Intervention \\
\hline Outpatient appointment & $£ 874$ & $£ 784$ \\
GP appointment & $£ 50$ & $£ 32$ \\
Combined other & $£ 101$ & $£ 58$ \\
Physiotherapist & $£ 34$ & Not applicable \\
Counsellor & $£ 41$ & Not applicable \\
Outpatient hospice & Not applicable & $£ 214$ \\
DTU Attendance & Not applicable & $£ 727$ \\
Total expected cost & $£ 1,100$ & $£ 1,775$ \\
\hline
\end{tabular}

a threshold value of $£ 20,000$ per QALY. At nine months the intervention is cost-effective in $50.2 \%$ of simulations and at 12 months $60.8 \%$ of simulations at a threshold value of $£ 20,000$.

\section{Discussion}

We report details of a full cost-utility analysis and associated sensitivity analyses of a rehabilitation intervention that has been shown to be clinically effective in a singlecentre randomised controlled trial. Our results demonstrate two things. First, that the results of the analysis are sensitive to the method used to estimate QALYs. This is well supported in the literature (for a discussion of this, see Manca and colleagues [30]). Second, it is also clear that the longer treatment benefit is maintained, the more likely it becomes that the intervention (which is provided over a short period of time) represents a costeffective use of resources. The CEACs in Figures 1 and 2 are used to show in what proportion of the Monte-Carlo simulations the intervention is expected to have a greater net-benefit compared with control across a range of threshold values of the decision maker's willingness to pay for a QALY [31]. In England, for the NHS NICE typically use a threshold range of $£ 20,000$ to $£ 30,000$ per QALY when determining whether a treatment is costeffective use of resources.

Our results suggest that a standardised hospice based day therapy intervention has the potential to be costeffective, though in the base-case analysis this effect was marginal with respect to the NICE threshold value of
$£ 20,000$ per QALY in England [32]. We have shown that when QALYs are estimated using the more robust areaunder-the-curve or baseline-adjusted approaches then the intervention is less likely to be cost-effective at three months than in our initial crude analysis. However, if the benefit of the intervention were maintained as intended following discharge from the service, then the likelihood of the intervention being cost-effective clearly increases; as expected, the longer any observed benefit is maintained post-treatment, the more likely the intervention is a cost-effective use of resources. This finding is independent of the method used to estimate QALYs.

Although an RCT provides a robust causal estimate of the intervention effect, a single trial may not provide sufficient evidence on which to base a decision on cost effectiveness [33]. For example, trials such as this which are limited to single centres, small sample sizes or restrictions on clinical diagnoses such as cancer types may not show to what extent the effectiveness of an intervention is transferable to other settings or patient populations. In addition trial designs, such as wait-list control, may not include an appropriate time horizon for the measurement of treatment costs and outcomes, and may not be designed to consider all relevant treatment alternatives [33]. Such limitations are often pragmatic and reflect funding restrictions or clinical circumstances. In this case, caution was exercised due to the ethics of withholding a potentially beneficial supportive intervention for those with a limited prognosis.

\section{Transferability of results}

In addition to the consideration of local factors and context that influence treatment outcomes in a single centre study, interpreting economic results also requires consideration of patterns of resource use and costs associated with a single centre. We have tried within this analysis to improve the transferability of the results to the wider NHS and other care providers by estimating costs using nationally reported unit costs and accounting for potential variation in costs to different providers. However, it was necessary to use some estimates of costs taken from the single delivery site. Local variation in practice, for example referral rates to secondary care or procedures for avoiding inpatient admissions, can greatly

Table 5 Base-case results (with Bayesian credible intervals)

\begin{tabular}{|c|c|c|c|c|c|c|c|}
\hline \multirow[b]{2}{*}{ Base-case } & \multicolumn{2}{|l|}{ Control } & \multicolumn{2}{|c|}{ Intervention } & \multicolumn{3}{|c|}{ Incremental } \\
\hline & Cost & QALYs & Cost & QALYs & Cost & QALYs & ICER \\
\hline & $(95 \% \mathrm{Cl})$ & $(95 \% \mathrm{Cl})$ & $(95 \% \mathrm{Cl})$ & $(95 \% \mathrm{Cl})$ & $(95 \% \mathrm{Cl})$ & $(95 \% \mathrm{Cl})$ & $\begin{array}{l}£ s / \\
\text { QALY }\end{array}$ \\
\hline \multirow{2}{*}{$\begin{array}{l}\text { Benefit as measured at three } \\
\text { months }\end{array}$} & $£ 1,193$ & 0.112 & $£ 1,928$ & 0.164 & $£ 735$ & 0.052 & $£ 14,231$ \\
\hline & $\begin{array}{l}(£ 840 \text { to } \\
£ 1,638)\end{array}$ & $\begin{array}{l}(0.102 \text { to } \\
0.123)\end{array}$ & $\begin{array}{l}(£ 1,481 \text { to } \\
£ 2,455)\end{array}$ & $\begin{array}{l}(0.159 \text { to } \\
0.168)\end{array}$ & $\begin{array}{l}(£ 221 \text { to } \\
£ 1,271)\end{array}$ & $\begin{array}{l}(0.040 \text { to } \\
0.063)\end{array}$ & \\
\hline
\end{tabular}


Table 6 Area under the curve scenario analysis (with Bayesian credible intervals)

\begin{tabular}{|c|c|c|c|c|c|}
\hline \multirow{3}{*}{ Area under the curve analysis } & \multicolumn{2}{|l|}{ Control } & \multirow{3}{*}{$\begin{array}{l}\text { Intervention } \\
\text { Cost } \\
(95 \% \mathrm{Cl})\end{array}$} & \multicolumn{2}{|l|}{ ICER } \\
\hline & Cost & QALYs & & QALYs & Es/ \\
\hline & $(95 \% \mathrm{Cl})$ & $(95 \% \mathrm{Cl})$ & & $(95 \% \mathrm{Cl})$ & QALY \\
\hline \multirow[t]{2}{*}{ Benefit as measured at three months } & $£ 1,202$ & 0.120 & $£ 1,936$ & 0.156 & $£ 20,514$ \\
\hline & ( $£ 842$ to $£ 1,631$ ) & (0.077 to 0.160$)$ & $(£ 1,483$ to $£ 2,459)$ & (0.136 to 0.176$)$ & \\
\hline \multirow[t]{2}{*}{ Benefit maintained to six months } & $£ 1,192$ & 0.231 & $£ 1,931$ & 0.312 & $£ 8,371$ \\
\hline & ( $£ 837$ to $£ 1,630$ ) & (0.138 to 0.318$)$ & $(£ 1,471$ to $£ 2,477)$ & (0.267 to 0.355$)$ & \\
\hline \multirow[t]{2}{*}{ Benefit maintained to nine months } & $£ 1,196$ & 0.344 & $£ 1,931$ & 0.470 & $£ 5,224$ \\
\hline & ( $£ 832$ to $£ 1,626)$ & (0.199 to 0.475$)$ & $(£ 1,481$ to $£ 2,460)$ & (0.398 to 0.537$)$ & \\
\hline \multirow[t]{2}{*}{ Benefit maintained to one year } & $£ 1,198$ & 0.454 & $£ 1,931$ & 0.629 & $£ 3,815$ \\
\hline & ( $£ 843$ to $£ 1,623$ ) & (0.247 to 0.623 ) & ( $£ 1,476$ to $£ 2,469)$ & (0.526 to 0.718$)$ & \\
\hline
\end{tabular}

influence the cost of providing an intervention. Analysis of data from a larger multi-centre trial would help address these limitations.

\section{Benefits over time}

Often the pragmatic design of clinical trials limits the follow-up period for data collection. The most-appropriate duration of follow-up for clinical outcomes may differ from those outcomes needed to estimate the long-term cost-effectiveness of an intervention, even if the observed cost-effectiveness ratio is trending towards that of the long-term ICER [34]. It would be ideal, though usually impractical, to measure any benefits accrued over the lifetime of the participant. In reality, assumptions about longer term outcomes must usually be made.

In this trial, the three month follow-up period and the wait list design were enforced for ethical reasons and data collected are unlikely to reflect the overall true outcomes for participants with respect to health related quality of life. It is also unlikely that those who experienced benefit at the end of the study period will lose the entirety of that benefit the moment treatment stops. What is not known is for how long benefit is maintained and at what rate it is lost (if at all) over

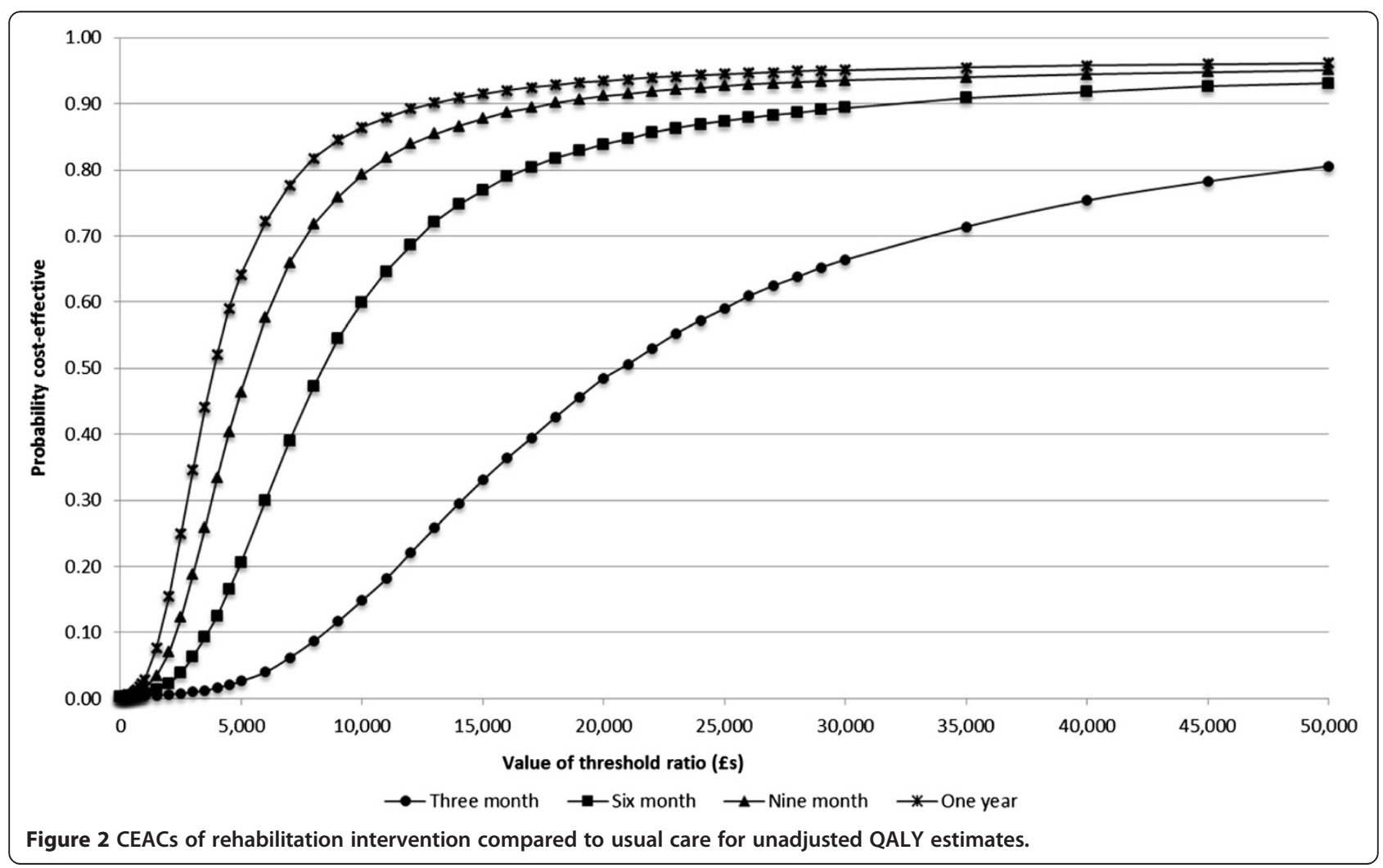


Table 7 Baseline adjusted scenario analysis (with Bayesian credible intervals)

\begin{tabular}{|c|c|c|c|c|c|}
\hline \multirow[b]{3}{*}{ Baseline adjusted analysis } & \multicolumn{2}{|l|}{ Costs } & \multirow{2}{*}{\multicolumn{2}{|c|}{$\begin{array}{l}\text { Estimated QALY } \\
\text { difference }\end{array}$}} & \multirow{3}{*}{$\begin{array}{l}\text { ICER } \\
\text { £s/QALY }\end{array}$} \\
\hline & \multicolumn{2}{|l|}{$(95 \% \mathrm{Cl})$} & & & \\
\hline & Control & Intervention & Mean & SE & \\
\hline \multirow[t]{2}{*}{ Benefit as measured at three months } & $£ 1,203$ & $£ 1,938$ & 0.008 & 0.009 & $£ 94,748$ \\
\hline & (£865 to $£ 1,615$ ) & ( $£ 1,540$ to $£ 2,394)$ & - & - & \\
\hline \multirow[t]{2}{*}{ Benefit maintained to six months } & $£ 1,202$ & $£ 1,931$ & 0.023 & 0.027 & $£ 29,835$ \\
\hline & ( $£ 837$ to $£ 1,658$ ) & ( $£ 1,471$ to $£ 2,437)$ & - & - & \\
\hline \multirow[t]{2}{*}{ Benefit maintained to nine months } & $£ 1,207$ & $£ 1,932$ & 0.039 & 0.046 & $£ 18,771$ \\
\hline & ( $£ 868$ to $£ 1,626)$ & $(£ 1,534$ to $£ 2,389)$ & - & - & \\
\hline \multirow[t]{2}{*}{ Benefit maintained to one year } & $£ 1,205$ & $£ 1,933$ & 0.054 & 0.064 & $£ 13,400$ \\
\hline & (£864 to $£ 1,611$ ) & ( $£ 1,540$ to $£ 2,389)$ & - & - & \\
\hline
\end{tabular}

time. In those with advanced cancer, these factors are likely to be influenced by familiarity with the hospice out-patient service, confidence in the probable responsiveness of the clinical team should the patient represent to the service and deterioration in clinical condition. We have attempted to address this through a series of scenario analyses that extend treatment benefit to a maximum of one year. Though this approach has obvious limitations based on the nonavailability of data, it provides decision makers with information derived from modelling scenarios likely to occur beyond the three month trial analysis period and may aid them in considering whether or not to implement the intervention.

\section{Alternative treatment options}

An additional limitation of the trial from the perspective of the economic analyst is that it compares only two alternative treatment modalities. In practice, the particular form of the intervention as practised in the trial is not the only possible way to organise and deliver rehabilitation services for patients living with advanced cancer. In determining the effectiveness from a clinical standpoint, this is appropriate. However it reduces the information

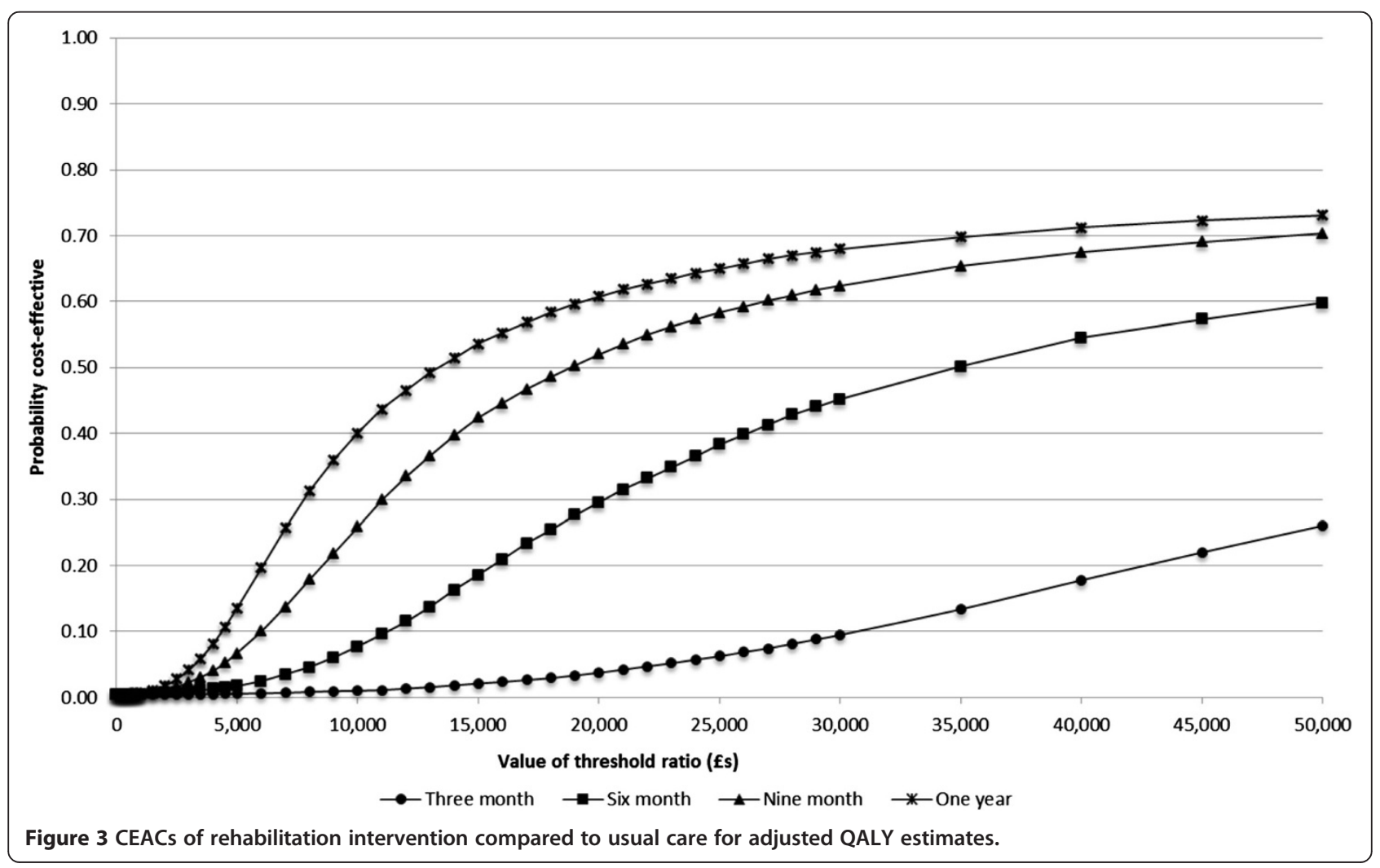


available to decision makers who must determine not just whether to offer rehabilitation services but how. Ideally, any future economic evaluation would examine not just the results of this trial but also include evidence from any other trials or sources of robust evidence of day therapy rehabilitation services. However, no other such services have been tested in an RCT, nor do any services have robust evidence of effectiveness and so at present, this evaluation represents the best possible evidence available to decision makers. In Table 4 we provide some evidence of the expected mean cost for each category of resource used in the analysis. Service planners may be able to use such evidence to develop local day therapy rehabilitation services.

\section{Research considerations for economic evaluations}

Caution is required when comparing individual resource use as in Table 4, as the study population was not sufficiently large to make such comparisons, nor was resource use a designated outcome of the trial. Any larger multi-centre study of this intervention should examine such potential resource use related outcomes for participants. It may be useful to explore which component of the intervention provides the most benefits for its cost. In practice, for such complex interventions the individual effect of each component may not be evaluable separately from the overall effect. In a larger study it would be possible to explore whether there are participant subgroups which might be more likely to benefit from the intervention. An exploratory analysis of our results suggests that women and those with lymphoma reported more benefits than other participants, although these findings are limited by a small sample size.

Another potential concern in economic evaluations is the risk of bias arising from the use of patient recall methods for collecting data on service use. Patient recall methods are commonly used to collect data on patient service use as part of clinical trials and other prospective studies. Concern exists that patients may not be able to recall with sufficient accuracy their contacts with health services over time [35]. Although the evidence base remains limited [35], some research has shown that recall periods of up to six months accurately capture patient resource use [27] The primary alternative to recall methods is the use a patient diary in which study participants record health service use on an on-going basis during the trial period. However, this method is not typically recommended, as respondent burden is considered high and the rates of completion are often low [36].

\section{Challenges for health care providers}

Both the range of ICERs and the CEAC for the basecase analysis indicate uncertainty for decision makers who must choose whether or not the intervention is a cost-effective use of scarce NHS resources. The withintrial analysis, based on costs and outcomes measured at three months, shows that the intervention sits on the margins of cost-effectiveness. This leaves decision makers with a good deal of uncertainty - it is as likely as not that the intervention is cost-effective if there is a willingness to pay of $£ 20,000$ per QALY. When the length of time that recipients might expect to maintain benefit from the intervention is extended the ICER decreases, though the probability that the intervention is cost-effective increase only slightly. This reflects the considerable degree of uncertainty in the trial data resulting from the small sample size.

When there is such uncertainty over the likely costeffectiveness of an intervention decision makers are faced with the question of whether or not to implement the intervention [37]. A number of factors beyond the ICER and CEAC results can influence this decision. These might include the ways in which a technology or intervention is delivered, what recipient groups are likely to be affected by adoption and questions of equity - that is, whether any recipient groups might be unfairly disadvantaged by the decision to adopt an intervention $[38,39]$. In the case of the intervention studied here, decision makers may be helped by further data on the value of treatment to recipient sub-groups and the ways in which the intervention can be delivered in different settings. In addition decision makers must consider the irreversibility of their decision and the consequences of a wrong decision [40]. In the case of a day therapy rehabilitation service, the establishment of such a service is likely to have few irreversible consequences in circumstances where capital investment requirements are likely to be low and staff training requirements minimal as specialist multi-disciplinary practitioners are usually available within hospice settings. Services could be reduced easily if further research shows that treatment is not cost-effective and resources redirected with relative ease.

\section{Conclusion}

Our analyses reveal uncertainty about whether the rehabilitation intervention represents a cost-effective use of health care resources when compared with usual care. Although commissioners may wish to consider introducing such day therapy services in view of the clinical benefits to patients, they should be mindful of the need for further evaluation of cost-effectiveness. This should be weighed against a) the potential benefits to recipients and b) the relative ease with which a decision could be reversed if the model used follows closely that used in the RCT on which this evaluation is based [30]. Where a day therapy service is introduced formal evaluation should be considered. Value of information analysis would help 
determine the benefits and inform the design of such an evaluation.

\section{Competing interests}

The authors declare that they have no competing interests.

\section{Authors' contributions}

All authors contributed to the conception of the study and the analysis plan. The analysis was conducted by JR with support from BL. LJ provided clinical insight for developing the model. All authors contributed to the preparation of the manuscript and had approval over the submitted manuscript.

\section{Authors' information}

All authors' posts were supported by Marie Curie Cancer Care core grant funding to the Marie Curie Palliative Care Research Unit, Department of Mental Health Sciences, University College London at the time of the study. The funders had no role in the conception or preparation of, or decision to submit, this manuscript.

\section{Acknowledgements}

The authors would like to thank Amanda Holman for her contributions to the development of the original study and comments on this manuscript.

Received: 21 January 2014 Accepted: 24 October 2014

Published online: 19 November 2014

\section{References}

1. Hodgkinson K, Butow P, Hunt GE, Pendlebury S, Hobbs KM, Wain G: Breast cancer survivors' supportive care needs 2-10 years after diagnosis. Support Care Cancer 2007, 15:515-523.

2. Mellon S, Northouse LL, Weiss LK: A population-based study of the quality of life of cancer survivors and their family caregivers. Cancer Nurs 2006, 29:120-131.

3. Greer JA, Jackson VA, Meier DE, Temel JS: Early integration of palliative care services with standard oncology care for patients with advanced cancer. CA Cancer J Clin 2013, 63:349-363.

4. Parikh RB, Temel JS: Early specialty palliative care. N Engl J Med 2014 370:1075-1076.

5. Lund S, Stone PC: Predicting survival in advanced cancer. Eur J Palliat Care 2013, 20:58-61.

6. National Council for Hospice and Specialist Palliative Care Services: Fulfilling lives: Rehabilitation in Palliative Care. London: National Council for Hospice and Specialist Palliative Care Services; 2000.

7. Stevens E, Jackson S, Milligan S: Palliative Nursing in the 21st Century. UK: Blackwell; 2014.

8. Roulston A, Bickerstaff D, Haynes T, Rutherford L, Jones $L$ : A pilot study to evaluate an outpatient service for people with advanced lung cancer. Int J Palliat Nurs 2012, 18:225-233.

9. Davies E, Higginson IJ: Systematic review of specialist palliative day-care for adults with cancer. Support Care Cancer 2005, 13:607-627.

10. Bradley SE, Frizelle D, Johnson M: Why do health professionals refer individual patients to specialist day hospice care? J Palliat Med 2011 14:133-138.

11. de Vries K, Wells J, Plaskota M: Evaluation of an admission and discharge process for specialist palliative day hospice in the United Kingdom using case study methodology. Int J Palliat Nurs 2012, 18:275-281.

12. Douglas HR, Normand CE, Higginson IJ, Goodwin DM: A new approach to eliciting patients' preferences for palliative day care: the choice experiment method. J Pain Symptom Manage 2005, 29:435-445.

13. Goodwin DM, Higginson IJ, Myers K, Douglas HR, Normand CE: Effectiveness of palliative day care in improving pain, symptom control, and quality of life. J Pain Symptom Manage 2003, 25:202-212.

14. Douglas HR, Normand CE, Higginson IJ, Goodwin DM, Myers K: Palliative day care: what does it cost to run a centre and does attendance affect use of other services? Palliat Med 2003, 17:628-637.

15. Mosoiu D, Dumitrescu M, Connor SR: Developing a costing framework for palliative care services. J Pain Symptom Manage 2014, 48(4):719-729.

16. Jones L, FitzGerald G, Leurent B, Round J, Eades J, Davis S, Gishen F, Holman A, Hopkins K, Tookman A: Rehabilitation in advanced, progressive, recurrent cancer: a randomized controlled trial. J Pain Symptom Manage 2013, 46:315. -U163.
17. Bonevski B, Sanson-Fisher R, Girgis A, Burton L, Cook P, Boyes A: Evaluation of an instrument to assess the needs of patients with cancer. Supportive Care Review Group. Cancer 2000, 88:217-225.

18. Craig P, Dieppe P, Macintyre S, Michie S, Nazareth I, Petticrew M: Developing and evaluating complex interventions: the new medical research council guidance. BMJ 2008, 337:a1655.

19. Dolan P: Modeling valuations for EuroQol health states. Med Care 1997, 35:1095-1108.

20. National Institute for Health and Clinical Excellence: Guide to the Methods of Technology Appraisal. London: National Institute for Health and Clinical Excellence; 2008

21. Round J: Is a QALY still a QALY at the end of life? J Health Econ 2012, 31(3):521-552

22. Morris S, Devlin N, Parkin D: Economic Analysis in Health Care. 1st edition. Chichester: John Wiley and Sons, Ltd; 2007.

23. O'Hagan A, Stevens JW: A framework for cost-effectiveness analysis from clinical trial data. Health Econ 2001, 10:303-315.

24. O'Hagan A, Stevens JW, Montmartin J: Bayesian cost-effectiveness analysis from clinical trial data. Stat Med 2001, 20:733-753.

25. Stevens JW, O'Hagan A: Incorporation of genuine prior information in cost-effectiveness analysis of clinical trial data. Int J Technol Assess Health Care 2002, 18:782-790.

26. Briggs $\mathrm{AH}$ : A Bayesian approach to stochastic cost-effectiveness analysis. An illustration and application to blood pressure control in type 2 diabetes. Int J Technol Assess Health Care 2001, 17:69-82.

27. Patel A, Rendu A, Moran P, Leese M, Mann A, Knapp M: A comparison of two methods of collecting economic data in primary care. Fam Pract 2005, 22:323-327.

28. Lesley C: Unit Costs of Health and Social Care. Cantebury: Personal Social Services Research Unit, University of Kent; 2011.

29. Department of Health: NHS Payment by Results 2010-11 National Tariff Information. 2010.

30. Manca A, Hawkins N, Sculpher MJ: Estimating mean QALYs in trial-based cost-effectiveness analysis: the importance of controlling for baseline utility. Health Econ 2005, 14:487-496.

31. Fenwick E, Byford S: A guide to cost-effectiveness acceptability curves Br J Psychiatry 2005, 187:106-108.

32. National Institute for Health and Clinical Excellence: Appraising Life-Extending, End of Life Treatments. London: National Institute for Clinical Excellence; 2009.

33. Sculpher MJ, Claxton K, Drummond M, McCabe C: Whither trial-based economic evaluation for health care decision making? Health Econ 2006, 15:677-687.

34. Glick H, Doshi J, Sonnad S, Polsky D: Economic Evaluation in Clinical Trials. 1st edition. Oxford: Oxford University Press; 2007.

35. Thorn JC, Coast J, Cohen D, Hollingworth W, Knapp M, Noble SM, Ridyard C, Wordsworth S, Hughes D: Resource-use measurement based on patient recall: issues and challenges for economic evaluation. Appl Health Econ Health Policy 2013, 11:155-161.

36. Sleed M, Eccleston C, Beecham J, Knapp M, Jordan A: The economic impact of chronic pain in adolescence: methodological considerations and a preliminary costs-of-illness study. Pain 2005, 119:183-190.

37. Claxton K, Posnett J: An economic approach to clinical trial design and research priority-setting. Health Econ 1996, 5:513-524.

38. Edlin R, Round J, McCabe C, Sculpher M, Claxton K, Cookson R: CostEffectiveness Analysis and Ageism: A Review of The Theoretical Literature. 2008

39. National Institute for Health and Clinical Excellence: Social Value Judgements: Principles for the Development of NICE Guidance. London: NICE; 2008.

40. Briggs A, Calxton K, Sculpher M: Decision Modelling for Health Economic Evaluation. 1st edition. Oxford: Oxford University Press; 2006.

\section{doi:10.1186/s12913-014-0558-5}

Cite this article as: Round et al:: A cost-utility analysis of a rehabilitation service for people living with and beyond cancer. BMC Health Services Research 2014 14:558 